\title{
Here today, gone tomorrow: biodegradable soft robots
}

\author{
Jonathan Rossiter*ac ${ }^{\text {ac }}$ Jonathan Winfield ${ }^{\text {bc }}$, Ioannis Ieropoulos ${ }^{\text {bc }}$ \\ ${ }^{a}$ Dept. of Engineering Mathematics, University of Bristol; ${ }^{b}$ Bristol Bioenergy Centre, University of \\ the West of England; ${ }^{\mathrm{c}}$ Bristol Robotics Laboratory
}

\begin{abstract}
One of the greatest challenges to modern technologies is what to do with them when they go irreparably wrong or come to the end of their productive lives. The convention, since the development of modern civilisation, is to discard a broken item and then procure a new one. In the 20th century enlightened environmentalists campaigned for recycling and reuse (R\&R). R\&R has continued to be an important part of new technology development, but there is still a huge problem of non-recyclable materials being dumped into landfill and being discarded in the environment. The challenge is even greater for robotics, a field which will impact on all aspects of our lives, where discards include motors, rigid elements and toxic power supplies and batteries. One novel solution is the biodegradable robot, an active physical machine that is composed of biodegradable materials and which degrades to nothing when released into the environment. In this paper we examine the potential and realities of biodegradable robotics, consider novel solutions to core components such as sensors, actuators and energy scavenging, and give examples of biodegradable robotics fabricated from everyday, and not so common, biodegradable electroactive materials. The realisation of truly biodegradable robots also brings entirely new deployment, exploration and bio-remediation capabilities: why track and recover a few large non-biodegradable robots when you could speculatively release millions of biodegradable robots instead? We will consider some of these exciting developments and explore the future of this new field.
\end{abstract}

Keywords: biodegradable robotics, biodegradable electroactive polymers, soft robotics, bio-remediation

\section{INTRODUCTION}

Modern society is a massive consumer of resources. Every plastic bag, every iPhone, every car depletes world resources. Fuels for internal combustion engines, plastics and many organic chemicals are directly derived from nonrenewable fossil fuels. Semiconductor components and electronic circuits use significant quantities of rare and exotic materials [1]. Even the gold used to coat most electrical contacts is a pressured resource. Exceptionally a vital material may be in abundance, such as silicates for silicon wafers, but typically these need to be mixed or joined to rare materials (such as semiconductor dopants) to realise their true functional value. The pressure on natural resources is huge and it is striking how soon the modern world is heading for critical events. While 'peak oil' - the point where no more fossil fuels can be discovered and resources will start to fall rapidly - has been talked about for decades, the notion of resource peaking is due to hit many other vital materials [2]. Take for example the lithium polymer battery, a staple of modern portable electronic devices and the focus of new and well-publicised initiatives to join solar technologies to in-home electrical storage [3]. The planetary supply of lithium is finite and these new technologies are putting an exponentially increasing demand on the raw material. The same is true, to greater or lesser extent for more mundane materials like copper and rare earth metals like neodymium, both important elements in conventional robotics and electromagnetics.

*Jonathan.Rossiter@bris.ac.uk, +44 117 3315601,www.bris.ac.uk/engineering/people/jonathan-m-rossiter

Electroactive Polymer Actuators and Devices (EAPAD) 2016, edited by Yoseph Bar-Cohen, Proc. of SPIE Vol. 9798, 97981S · C 2016 SPIE · CCC code: 0277-786X/16/\$18 · doi: 10.1117/12.2220611 
The pressure of this consumption has led to research into the three R's of Reduce, Reuse, Recycle. Unfortunately this will only delay the resource depletion described above: Any reduction in use per device is counteracted by an exponential increase in device consumption; Reuse of end-user products is largely ignored as they are built with finite lifetimes in mind; Recycling of materials can be extremely difficult - recovering rare elements from semiconductors for example is almost impossible given their low volumes and extensive packaging.

The solution may be far more radical than the three R's. If we can target our research to develop renewable replacements for currently non-renewable technologies we may be able to reach a sustainable and stable state where resource peaking can be forestalled indefinitely. One area where this has high potential is in soft robotics. In contrast to conventional robotics which uses rigid materials and electromagnetic drives soft robotics re-writes the design and fabrication rule book. This is a major opportunity to develop truly sustainable soft robotics. The most promising of soft robotics technologies that can deliver this potential are smart materials. These can be made from benign and readily available materials, thus reducing resource pressures, and can even be made to degrade safely within the environment, yielding the potential for total energy and resource recycling.

In this paper we will examine the motivation and reality of designing and implementing totally biodegradable and environmentally safe robots. We propose technologies with the greatest potential for further development and examine how the 'throw-away' robot can change the way we think about robotics as a whole.

\section{DEATH AND DECAY IN NATURE}

The natural environment is a well-balanced machine. It operates as a closed system in which new organisms are born, live and die. The raw materials that make up these dead organisms are reused within the system either directly, through digestion by other organisms higher up the food chain, or by gradual decay into constituent molecules that then enter the food chain near the bottom. There is therefore a range of particulate sizes that decay and breakdown. In Nature this is not a problem because niche organisms have evolved to use materials at all particulate sizes.

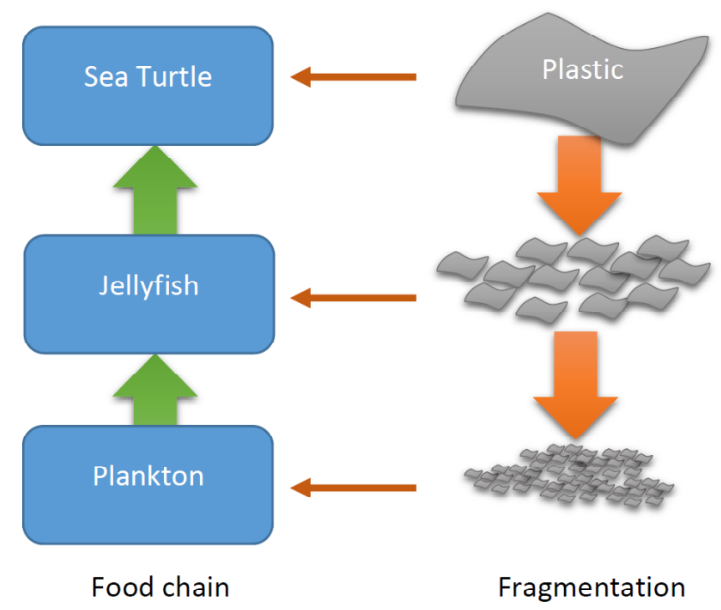

Figure 1. Effect of plastic decomposition and fragmentation on the food chain

The degradation of man-made material, on the other hand, does not result in uniformly benign effects. Take for example the degradation of a plastic bag. 100 billion units are consumed in the EU alone each year and a significant number of these end up released, by accident or design, into the environment. Non-biodegradable bags, such as those made from Polyethylene will take upwards of 500 years to decay and can have immediate impact on living creatures such as sea birds and turtles [4]. Even notionally biodegradable plastic bags are not as compatible with the natural environment as one would hope or expect [5]. Under photonic, chemical and mechanical effects these plastics typically fragment into 
smaller particles, which still retain many of their resilient properties (Figure 1). These fragments are digested by many organisms, depending on their size. Large pieces resemble the natural food of larger animals including turtles and when consumed can block their digestion. Microscopic pieces are consumed by plankton and other microorganisms where they are concentrated and then consumed by organisms higher up the food chain [6]. The degradation of materials covers a wide range of timescales from days to hundreds of years (over five orders of magnitude) as shown in Table 1.

Table 1. Biodegradation of common materials. Adapted from [7]

\begin{tabular}{|l|l|}
\hline Vegetables & 5 days -1 month \\
\hline Paper & $2-5$ months \\
\hline Cotton T-shirt & 6 months \\
\hline Orange peels & 6 months \\
\hline Tree leaves & 1 year \\
\hline Wool socks & $1-5$ years \\
\hline Plastic-coated paper milk cartons & 5 years \\
\hline Leather shoes & $25-40$ years \\
\hline Nylon fabric & $30-40$ years \\
\hline Tin cans & $50-100$ years \\
\hline Aluminium cans & $80-100$ years \\
\hline Glass bottles & 1 million years \\
\hline Styrofoam cup & 500 years to forever \\
\hline Plastic bags & 500 years to forever \\
\hline
\end{tabular}

Microbial degradation can occur in the presence (aerobic) or absence (anaerobic) of oxygen. In aerobic degradation aerobic bacteria (aerobes) degrade organic materials rapidly, generate no pungent gases, but do produce large amount of waste products. Aerobes include xanthomonas and comamonas. The active breakdown mechanisms includes enzymatic action to break down compounds into smaller chunks (simpler polymers) and respiration to break down simple polymers to basic elements and energy. Anaerobic degradation on the other hand operates in the absence of oxygen and employs hydrolysis, acidogenesis, acetogenesism and methanogenesis to break down organic materials. Anaerobes include claustridium and eubacterium spp. Anaerobic digestion is a slow process that generates pungent gases, but less waste is produced then aerobic degradation. Figure 2 shows stages of aerobic and anaerobic degradation for common naturallyoccurring organic polymers.

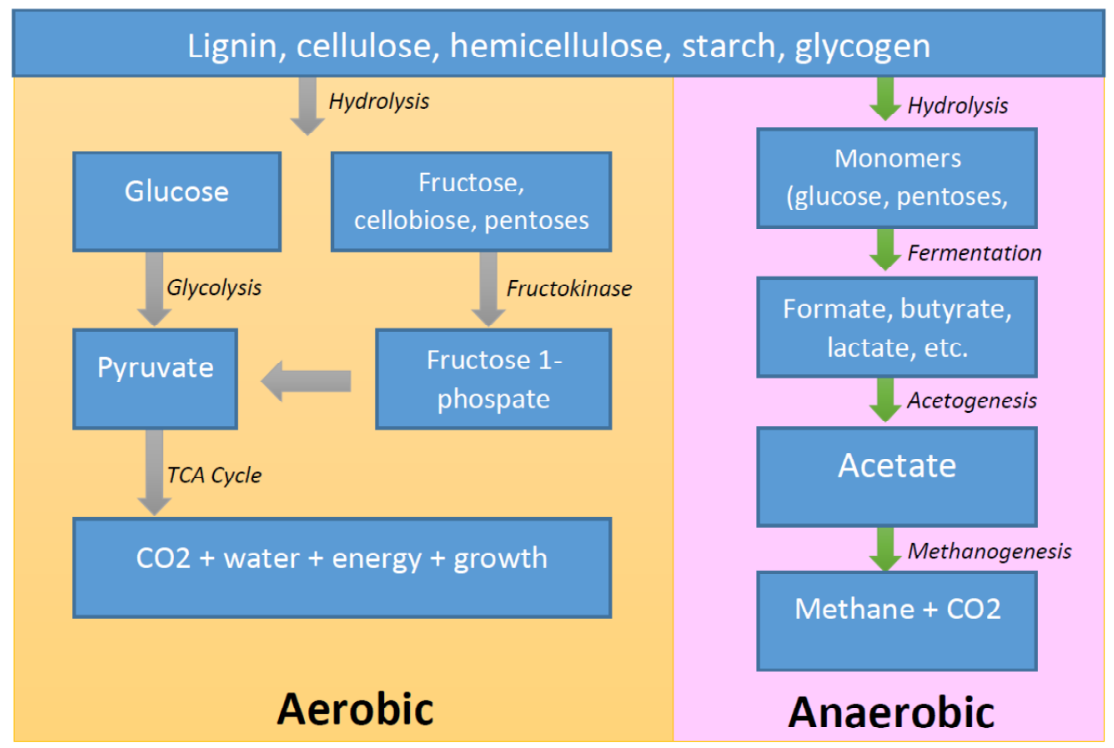

Figure 2. Stages of aerobic and anaerobic digestion of common organic polymers. Adapted from [8] 
A further advantage of natural organisms and the way they die and degrade is in the capture and storage of climactically influential chemicals. Microorganisms in the sea including zooplankton and phytoplankton breed in the warm surface waters and when they die they sink to the sea bed, carrying with them an important mass of carbon [9]. This carbon sequestration represents a significant contribution to the global carbon cycle. Mimicking this processes, or at least taking account of it in artificial systems, may help to reduce the effects of global climate change.

To take advantage of the efficiency and benefit of controlled death and degradation in the environment we ideally need to fuse directly into the natural food chain. This faces head-on the inevitability that any degradation of a man-made device will enter the food chain as natural organisms inject the fragmented materials. The materials used must therefore be totally compatible with the whole bio-system.

\section{BIOCOMPATIBLE AND BIODEGRADABLE POLYMERS}

Let us now consider the range of natural and synthetic polymers from which we may select building blocks for biodegradable robotics. It is informative to consider here not only biodegradability but the complimentary characteristic of biocompatibility. We can consider the two-dimensional space of biodegradability and biocompatibility as shown in Figure 3. We partition this space informally into biodegradable and non-biodegradable polymers and biocompatible and non-biocompatible polymers. Here we include all polymers including plastics, elastomers and biopolymers. Ideally we want to avoid polymers within region A and preferentially choose polymers from region B. Note that some polymers such as polysiloxanes are highly biocompatible (such as silastic used in medical implants [10]) but are not readily biodegradable. These are shown in region $\mathrm{C}$. We will also avoid polymers in region $\mathrm{D}$ because their biodegradability properties are out-weighed by their toxicity.

Examining materials within region B (biodegradable and non-toxic) we find almost exclusively biopolymers. These include naturally occurring materials such as collagen/gelatine, cellulose, and chitosan. We can also include all derived biological materials such as muscle fibres and rigid skeletal tissue (although strictly outside the remit of soft robotics). There are few, but notable, artificial plastic materials that fall into region B. These include polycaprolactone (PCL), a synthetic biodegradable polyester, and polylactic acid (PLA), a thermoplastic aliphatic polyester derived from renewable resources.

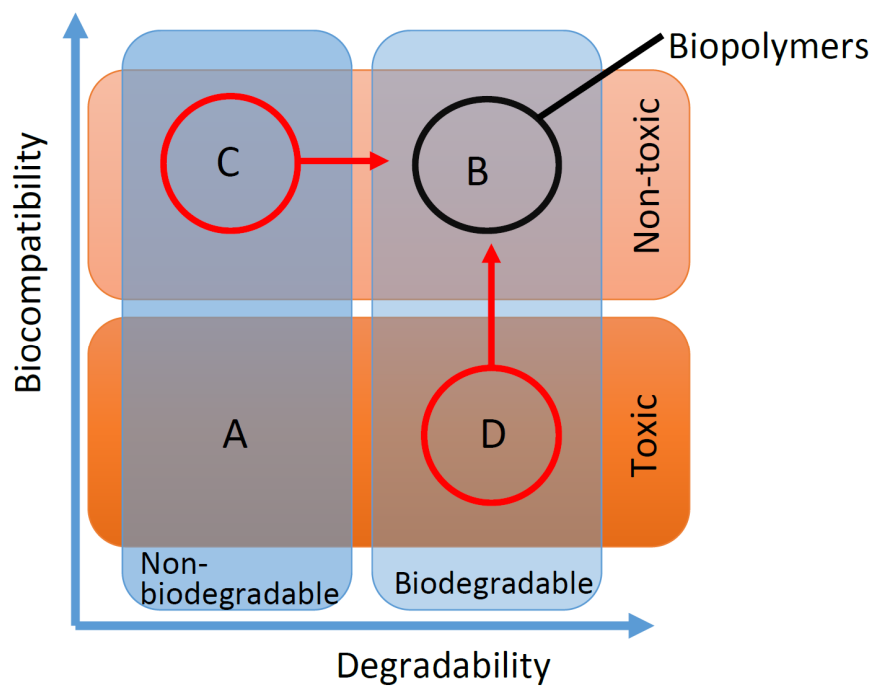

Figure 3. Broad classes of materials divided by toxicity and persistence. Red arrows show desired routes for achieving biodegradable and biocompatible materials from less capable materials. 
From Figure 3 we identify three options in selecting materials for biodegradable robots: 1 . We can use existing biopolymers such as cellulose and gelatine or existing biodegradable plastics such as PLA and PCL; 2. We can start with some non-biodegradable biocompatible polymer, such as PDMS, and try to make it biodegradable, or; 3. We can start with a toxic, but degradable polymer and attempt to make that non-toxic. The latter two options require research into polymer chemistry while the first option is the most readily applicable.

\section{ROBOT AS ORGANISM}

So far we have only considered the 'raw' polymers which could be used to make a biodegradable robot. These are, in their default state, non-active or non-smart materials. If we were to make a robot we might initially use these to form the robot body. Yet, a robot is more than a passive body. Rather, we can think of a robot as being made up of three core components: The body, the stomach and the brain. This is a natural, high level, decomposition of any organism, biological or artificial. The body is required to contain the organism and to provide means of locomotion and internal mechanisms (such as gut peristalsis or vascular control). The stomach provides a means of converting environmental energy into a form that the organism can use. The brain is the control system for the organism, in charge of homeostatic regulation, sensor and motor control, reasoning and goal-directed behaviour. Any robotic organism will have these three essential components.

Now let us focus on the body of the robot. The raw polymers described above can readily fulfil the role of a non-active body. They may be rigid (e.g. PLA) or soft (e.g. collagen) but they cannot, on their own, generate motion from stored energy. In order for the organism to move, these materials must be turned from non-smart materials to smart materials. In a similar fashion to the compatibility/biodegradation transformation described in Figure 3 for passive materials, there are two ways by which biodegradable smart materials can be realised: existing non-biodegradable smart materials can be converted into biodegradable form, or non-smart biodegradable materials can be made smart. We will consider the latter case in the next section.

\section{BIODEGRADABLE ELECTOACTIVE POLYMERS}

Electroactive polymers can be partitioned into two broad categories, electronic (including dielectric elastomer actuators) and ionic (including ionic polymer composites and conducting polymers). These two categories both transduce electrical energy into mechanical energy, but through different mechanisms. Dielectric elastomer actuators (DEAs) are large compliant capacitors that deform under Maxwell stresses induced by Coulomb forces between, and within, their compliant electrode [11]. Their fundamental construction is an insulating planar elastomer coated by conducting electrodes. Thus only two materials are needed for DEA fabrication. Ionic polymers are also planar structures consisting of a central membrane between conducting electrodes. The significant difference with ionic polymers is that the central membrane must be porous to all or selective ionic groups. Because the electric fields in ionic polymer actuators are much lower than DEAs $(\mathrm{kV} / \mathrm{m}$ for ionic polymers versus $\mathrm{MV} / \mathrm{m}$ for dielectric elastomers) the electrodes used in ionic polymers typically have higher conductivity than those used in DEAs. The base materials needed for these actuators are shown in Table 2, along with the contemporary non-biodegradable materials that are used for their fabrication.

Table 2. Typical materials used for electroactive polymer fabrication

\begin{tabular}{|l|l|l|}
\cline { 2 - 3 } \multicolumn{1}{c|}{} & Ionic & Electronic \\
\hline Central membrane & Nafion, Flemion, PVDF & $\begin{array}{l}\text { PDMS, Polyurethanes, } \\
\text { Polyacrylates }\end{array}$ \\
\hline Electrodes & $\begin{array}{l}\text { Gold, Platinum, Carbon } \\
\text { nanotubes }\end{array}$ & $\begin{array}{l}\text { Carbon grease, Silver, Implanted } \\
\text { gold, Carbon nanotubes }\end{array}$ \\
\hline
\end{tabular}


From Figure 3 we have shown that biopolymers are materials that are both non-toxic and biodegradable. In fact these materials are highly suited to use in electroactive polymer actuators. Biopolymers include materials that are elastomeric, dielectric and ion conducting, all potential substitutes for the materials shown in Table 2.

\subsection{Dielectric elastomer actuators}

The elastomer membrane in DEAs can be replaced by natural rubber (latex). Natural rubber has long been known to work in dielectric elastomer actuators [12] and dielectric elastomer generators (DEGs) [13]. The resilience of natural rubber (approx. $63 \%$ at room temp, rising to $80 \%$ at $115 \mathrm{C}$ ) can be an advantage, especially in DEGs. Recently ionic hydrogel mixtures have been shown to be extremely effective as DEA electrode materials $[14,15]$. Suitable hydrogels include gelatine, a natural and biodegradable biopolymer. The conductivity of these stable polymers is achieved through the inclusion of mobile ionic species, for example by mixing the gelatine with common salt $(\mathrm{NaCl})$ solution. There are further benefits of using biopolymers for electrodes, including the property of transparency [14].

Biodegradation of these polymers (natural rubber and gelatine) has been shown in [16] and Figure 4 shows a typical biodegradation test. Here collagen coupons were left to degrade in garden compost.

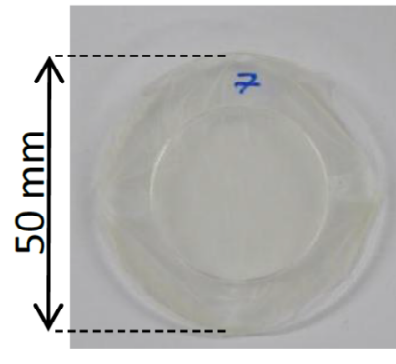

Day 0

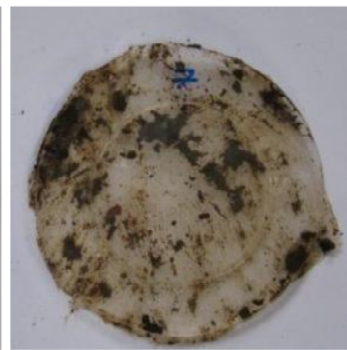

Day 4

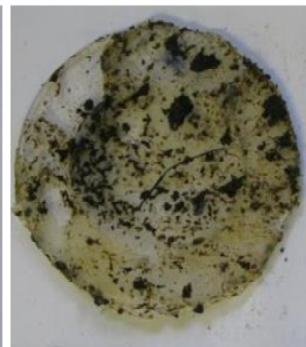

Day 11

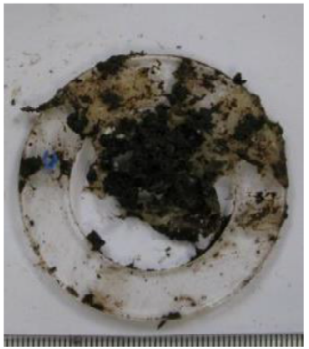

Day 18

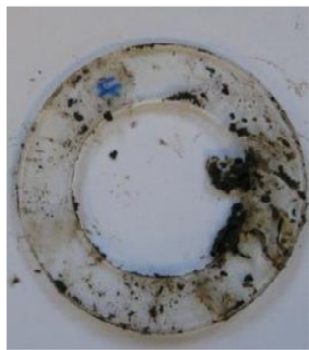

Day 25

Figure 4. Rapid decomposition of a collagen sample in outdoor compost heap

\subsection{Ionic polymer actuators}

Biopolymers for ionic actuators have been studied in some depth, but with little consideration of their biodegradability. The main emphasis has instead been on the low cost and free availability of the raw materials. For example the work of Kim et al. on EAP paper has sought to use cellulose materials (paper) for ionic actuators [17]. Likewise research into chitosan ionic actuators has shown the potential of these naturally biodegradable actuators [18]. More recently we have shown ionic actuation of biodegradable gelatine [19]. This actuation was driven by migration of ions within a $\mathrm{NaCl}$ salt solution between two metal electrodes (Figure 5). This further shows that commonly available biodegradable materials can be used for both the ionic electrolyte and electrode. It was also shown that such actuators can naturally dehydrate and remain in a 'hibernation state' with no decomposition. When subsequently hydrated they continued to work as actuators.

A large focus of recent research in ionic actuators has been on ionic liquids [20]. These liquids can replace the traditional aqueous electrolyte and, because they are highly stable in air, enable the actuator to operate outside water environments. Cholinium-based ionic liquids offer the most potential for biodegradability. Initial toxicity tests have shown good biocompatibility but the long term effects of the release of such ionic liquids in the environment is still unknown [21]. 

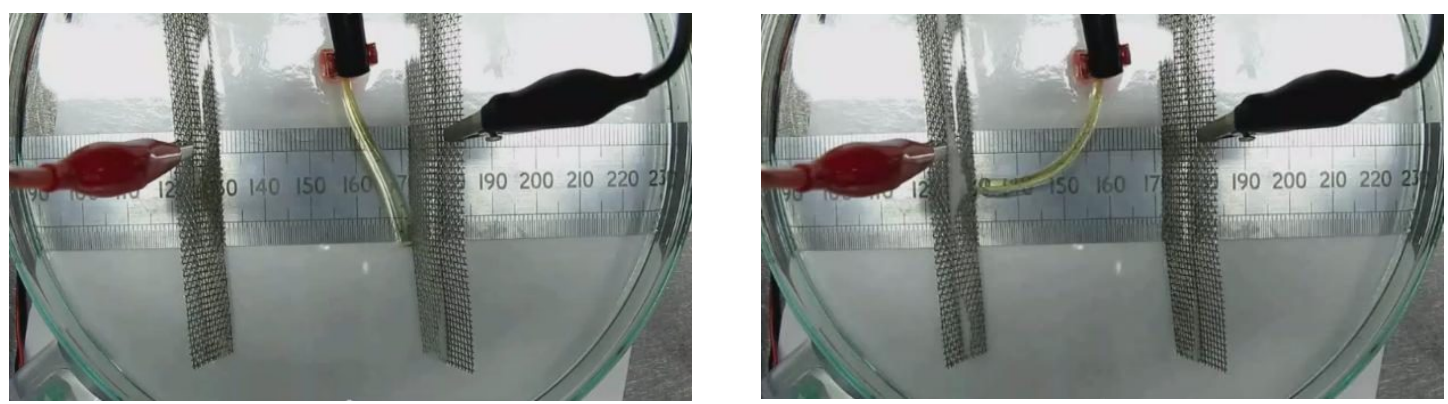

Figure 5. Bipolar electro-actuation of biodegradable gelatine actuator

\subsection{The power source for biodegradable robots}

For any robot to function a source of power, such as a battery, is essential. In terms of a biodegradable robot the unit supplying the energy and even the fuel should be biodegradable and sustainable. Microbial fuel cells (MFCs) employ anaerobic bacteria to transform the chemical energy in organic matter to electrical energy. If these were employed on a robot this electrical energy could then be transformed into mechanical energy, for locomotion perhaps, through electroactive polymers. For decades, MFCs have been constructed using rigid plastics, polymeric membranes and expensive toxic elements. Recently, however, less conventional, biodegradable materials have been successfully applied. Natural rubber has proven to be a viable substitute to conventional membranes over long term operation where the act of biodegradation actually aided performance [22]. Other materials such as paper [16], egg, gelatine, PLA and lanolin have all demonstrated their suitability as viable working components in MFCs [23]. The culmination of that work was the production of a stack of biodegradable MFCs capable of generating usable power, whilst utilising waste liquid such as urine as the fuel source.

\section{CURRENT AND FUTURE APPLICATIONS}

Biodegradable soft robots have potential to impact on all aspects of our daily life, from manufacturing to consumer devices and from medical treatments to wearable electronics. We highlight three high-impact applications:

\subsection{Bioremediation and environmental monitoring}

As industrial and agricultural activities have an increasing impact on environmental balance we require more sophisticated means of monitoring environmental pollution and of initiating one-site environmental clean-up. Pressing examples include harmful algal blooms resulting from nitrate run-off from agricultural land (Figure 6) and petrochemical spills in rivers and seas. Biodegradable robots offer the ideal solution to this challenge. These robots can be deployed on-site to monitor pollutant levels and to concentrate, treat and remove pollution. Unlike existing monitoring and remediation machines, which are large and few in number, biodegradable robots can be made in large numbers and speculatively released into the environment. In contrast to conventional non-biodegradable machines which must be constantly tracked and collected at the end of their operational life, biodegradable robots do not need to be tracked or recovered because they will degrade benignly in the environment. This capability changes the way we deploy robotics. Instead of deploying and recovering a small number of robots we can speculatively deploy hundreds, thousands or even millions of biodegradable robotics safe in the knowledge that they will do no harm to the environment. 


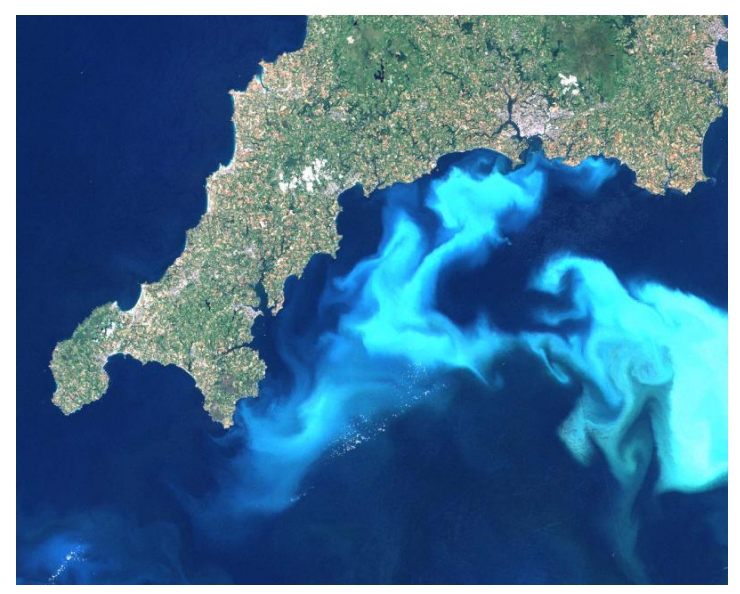

Figure 6. Algal bloom (light color) off the Cornish coast, UK, 1999 [Steve Groom, Plymouth Marine Laboratory]

\subsection{Disposable robotics and devices}

The consumeristic nature of modern developed society has led to a 'use and discard' mentality. To be compatible with this we can develop disposable robots and active assist and functional robotic devices that can be used and, once they are no longer able to operate, or are no longer fashionable, can be discarded with minimal or, potentially, positive environmental impact. These include all manner of household consumer objects from active-ergonomic tools to medical devices and treatments such as active wound dressings. It is to be expected that the pressure of this consumer-driven movement will be a major driver of this field in the next decade. In much the same way as biodegradable plastics have become common place in product packaging, we expect degradable and disposable robotic devices to be likewise ubiquitous. One can envisage even more everyday items such as food packaging to be made from active soft robotics they could bend and twist in our hands and impart more information about the contents and its properties than conventional packaging.

\subsection{Wearables/clothing}

One area where biodegradable soft robotic has large potential impact is in smart and active wearable devices. The combined markets for functional and aesthetic clothing are big drivers for technology development and soft robotics are placed to provide soft and compliant smart clothing and second-skins. When damaged or old these can be discarded and will degrade safely. The ultimate extension to this 'use and discard' practice is to have wearable and body-integrating soft robotic technologies that are worn once and discarded, much as disposable contact lenses have become common in ophthalmology.

\section{CONCLUSIONS}

In this paper we have briefly introduced and reviewed the field of biodegradable soft robotics. It is clear that there is great need for these technologies, particularly to meet the great challenges of environmental monitoring and remediation. On the other hand, the ability to use and discard soft robotics means that they will have great impact on ubiquitous immediate applications including wearables, fashion and active product packaging. We have also shown that the future of biodegradable soft robotics requires concerted effort in the chemistry of both biodegradable and smart materials. It is at the confluence of these two fields that the greatest progress in biodegradable soft robotics will be made. 


\section{ACKNOWLEDGEMENTS}

This work was supported by the Leverhulme Trust Project Grant RPG-362. Jonathan Rossiter is EPSRC Fellow (grant number EP/M020460/1) and also supported by the RoboSoft Coordination Action on Soft Robotics (FP7). Ioannis Ieropoulos is EPSRC Career Acceleration Fellow (grant numbers EP/I004653/1 and EP/L002132/1).

\section{REFERENCES}

[1] "Rare Earths Elements in High-Tech Industries: Market Analysis and Forecasts amid China's Trade" January 2016, 270 pages, Research and Markets (2016)

[2] Hubbert, M.K., "Techniques of Prediction as Applied to Production of Oil and Gas," US Department of Commerce, NBS Special Publication 631, (1982).

[3] Tesla Powerwall, 2016, http://www.teslamotors.com/powerwall (28 February 2016)

[4] Schuyler, Q.A., Wilcox, C., Townsend, K., Hardesty, B.D., Marshall, N.J., "Mistaken identity? Visual similarities of marine debris to natural prey items of sea turtles". BMC Ecol. 14: 14. (2014).

[5] Accinelli, C., Saccà, M.L., Mencarelli, M. and Vicari, A. "Deterioration of bioplastic carrier bags in the environment and assessment of a new recycling alternative" Chemosphere. 89 (2), pp.136-143. (2012)

[6] Wagner, M., Scherer C., et al. "Microplastics in freshwater ecosystems: what we know and what we need to know," Environmental Sciences Europe. 26, 2014, doi:10.1186/s12302-014-0012-7 (2014)

[7] Measuring Biodegradability, Sciencelearn.org.nz. http://sciencelearn.org.nz/Contexts/Enviro-imprints/LookingCloser/Measuring-biodegradability, (19 June 2008)

[8] Kaur, S., "Mechanism of aerobic \& an aerobic biodegradation," slideshare.net, www.slideshare.net/07sudha/mechanism-of-aerobic-an-aerobic-biodegradation (5 October 2014)

[9] Iglesias-Rodriguez M.D., Halloran P.R., Rickaby R.E.M., Hall I. R., Colmenero-Hidalgo E., et al. "Phytoplankton calcification in a high-CO2 world," Science 320: 336-340 (2008)

[10] Morgan S., Ng A., Clough T. "The long-term outcome of silastic implant arthroplasty of the first metatarsophalangeal joint: a retrospective analysis of one hundred and eight feet," International Orthopaedics. 2012;36(9):1865-1869. doi:10.1007/s00264-012-1576-8. (2012)

[11] Pelrine, R., Kornbluh, R., Pei, Q., Joseph, J. "High-Speed Electrically Actuated Elastomers with Strain Greater Than 100\%”. Science 287 (5454): 836-839. doi:10.1126/science.287.5454.836. (2000).

[12] Tangboriboon, N., Datsanae, S., Onthong, A., Kunanuruksapong, R., Sirivat, A., "Electromechanical responses of dielectric elastomer composite actuators based on natural rubber and alumina," Journal of Elastomers and Plastics March 2013 vol. 45 no. 2 143-161 (2013)

[13] Koh, S. J. A., Keplinger, C., Li, T., Bauer, S., and Suo, Z., "Dielectric Elastomer Generators: How Much Energy Can Be Converted?" in IEEE/ASME Transactions on Mechatronics, vol. 16, no. 1, pp. 33-41, (2011)

[14] Keplinger, C., Sun, J-Y., Foo, C.H., Rothemund, P., Whitesides, G.M., Suo, Z., "Stretchable, Transparent, Ionic Conductors," Science, 30 Aug 2013, Vol. 341, Issue 6149, pp. 984-987 (2013)

[15] Rossiter, J.M., Conn, A.T., Cerruto, A., Winters, A. and Roke, C., "Colour gamuts in polychromatic dielectric elastomer artificial chromatophores," Proceedings of SPIE - The International Society for Optical Engineering. SPIE (2014)

[16] Winfield, J., Chambers, L.D, Rossiter, J.M., Stinchcombe, A., Walter, X.A., Greenman, J., Ieropoulos, I. (2015a) "Fade to green: A biodegradable stack of microbial fuel cells" ChemSusChem 8 (16): 2705-2712

[17] Yun, S., Kim, J., Song, C., "Performance of Electro-active paper actuators with thickness variation," Sensors and Actuators A: Physical, Volume 133, Issue 1, 8 January 2007, Pages 225-230, (2007)

[18] Lu, L., Chen, W. "Biocompatible composite actuator: a supramolecular structure consisting of the biopolymer chitosan, carbon nanotubes, and an ionic liquid," Advanced Materials, 2010 Sep 1;22(33):3745-8. doi: 10.1002/adma.201001134. (2010)

[19] Chambers, L., Winfield, J., Ieropoulos, I. and Rossiter, J.M., "Biodegradable and edible gelatine actuators for use as artificial muscles". Proceeding of SPIE: Electroactive Polymer Actuators and Devices. SPIE - Int. Soc. Optical Engineering, Bellingham (2014) 
[20] Mukai, K., Asaka, K., Kiyohara, K., Sugino, T., Takeuchi, I., Fukushima, T., Aida, T., "High performance fully plastic actuator based on ionic-liquid-based bucky gel," Electrochimica Acta, Volume 53, Issue 17, 1 July 2008, Pages 5555-5562, (2008)

[21] Petkovic, M., Ferguson, J.L,. Gunaratne Nimal, H.Q., Ferreira, R., Leitao, M.C., Seddon, K.R., Rebelo, L.P.N., Pereira, C.S., "Novel biocompatible cholinium-based ionic liquids-toxicity and biodegradability," Green Chemistry, 12, 643-649 (2010)

[22] Winfield, J., Ieropoulos, I., Rossiter, J., Greenman, J. and Patton, D. "Biodegradation and proton exchange using natural rubber in microbial fuel cells", Biodegradation, 24: 733-739 (2013)

[23] Winfield, J., Chambers, L.D, Rossiter, J.M., Greenman, J., Ieropoulos, I. "Urine-activated origami microbial fuel cells to signal proof of life," Journal of Materials Chemistry A, Issue 13 vol 3., pp. 7058-7065, DOI: 10.1039/C5TA00687B, (2015) 